\title{
Depth of Muscularis Propria Invasion Does Not Prognosticate Survival in T2 Esophageal Adenocarcinoma
}

\author{
CHRISTOPHER W. SEDER ${ }^{1}$, BRETT MAHON ${ }^{2}$, MARK HENNON ${ }^{3}$, MATHEW THOMAS ${ }^{4}$, \\ CHARLES M. LEVEA ${ }^{5}$, KRISTINA A. MATKOWSKYJ ${ }^{6}$, MURLI KRISHNA ${ }^{7}$, \\ ROBERT MEDAIROS ${ }^{1}$, RYAN A. MACKE $^{8}$, SANJIB BASU $^{9}$ and JUSTIN D. BLASBERG ${ }^{10}$ \\ ${ }^{1}$ Department of Cardiovascular and Thoracic Surgery, ${ }^{2}$ Pathology, and \\ ${ }^{9}$ Preventative Medicine, Rush University Medical Center, Chicago, IL, U.S.A.; \\ ${ }^{3}$ Department of Thoracic Surgery, and ${ }^{5}$ Pathology, Roswell Park Cancer Institute, Buffalo, NY, U.S.A.; \\ ${ }^{4}$ Department of Surgery, Mayo Clinic, Jacksonville, FL, U.S.A.; \\ ${ }^{6}$ Department of Pathology and Laboratory Medicine, ${ }^{7}$ Pathology, and \\ ${ }^{8}$ Surgery, University of Wisconsin-Madison, Madison, WI, U.S.A.; \\ ${ }^{10}$ Department of Surgery, Yale University, New Haven, CT, U.S.A.
}

\begin{abstract}
Aim: Although tumor depth of invasion is strongly associated with risk of lymph node metastasis and long-term survival in patients with esophageal adenocarcinoma, the significance of differential T2 invasion (inner circular layer versus outer longitudinal layer) is unknown. The current study was undertaken to explore the hypothesis that greater T2specific depth of invasion is associated with inferior long-term outcomes in patients with esophageal adenocarcinoma treated with esophagectomy. Patients and Methods: Demographic, treatment, and outcome data were collected for patients with resected pT2NO-3MO esophageal adenocarcinoma treated between 2005 and 2015 pooled from four U.S. academic medical centers. Two blinded pathologists evaluated depth of muscularis propria tumor invasion. Univariate and Cox proportional hazard regression analyses were performed to identify prognostic factors for overall (OS) and disease-free (DFS) survival, and Kaplan-Meier analysis to compare survival differences specific to prognostic factors. Results: A total of 84 patients were identified for analysis (53 with circular invasion; 31 with longitudinal invasion), with a median age of 66 years. Sixty percent of patients (50/84)
\end{abstract}

This article is freely accessible online.

Presented: 53rd Annual Meeting of the Society of Thoracic Surgeons; January 21-25, 2017, Houston, Texas.

Correspondence to: Christopher W. Seder, MD, 1725 W. Harrison St, Suite 774; Chicago, IL 60612, U.S.A. Tel: +1 3129426642, Fax: +13127389763, e-mail: christopher_w_seder@rush.edu

Key Words: Esophageal cancer, adenocarcinoma, outcomes. received induction therapy prior to esophagectomy. The median OS and DFS was 58 months $(95 \%$ confidence interval $(C I)=42$ months-not reached) and 27 months $(95 \%$ CI=13.7-66 months) respectively. Depth of muscularis propria invasion did not correlate with $O S$ or DFS on univariate ( $p=0.42$; and $p=0.34$, respectively) or multivariate ( $p=0.15$ and $p=0.21$, respectively) analysis after adjustment for age, nodal status, perineural invasion, and tumor grade. These findings did not vary by induction therapy status. Conclusion: Depth of muscularis propria invasion does not appear to correlate with survival in patients with esophageal adenocarcinoma.

Esophageal cancer is the sixth leading cause of cancer death, with nearly 500,000 people diagnosed worldwide each year $(1,2)$. Despite ongoing clinical and laboratory efforts, the incidence of esophageal cancer continues to rise, with 5-year survival rates of only $15-25 \%$ (1-3). Esophageal squamous cell carcinoma remains the most common histological subtype, however, adenocarcinoma is becoming increasingly prevalent in North America and some European countries.

The optimal treatment strategy for patients with T2 esophageal cancer is controversial (4). Although esophageal ultrasound (EUS) has limited ability to assess depth of muscular invasion (4-7), pathological analysis can readily differentiate between circular and longitudinal muscle layer invasion. However, the seventh edition of the American Joint Committee on Cancer (AJCC) staging system currently does not account for depth of muscular invasion when risk stratifying patients with esophageal cancer (8). Recently, depth of muscularis propria tumor invasion has been shown to independently prognosticate survival in esophageal squamous cell carcinoma (9). 
Current National Comprehensive Cancer Network (NCCN) guidelines recommend that selected 'high-risk' patients with $\mathrm{T} 2 \mathrm{~N} 0$ esophageal adenocarcinoma who did not receive induction therapy and underwent $\mathrm{R} 0$ resection be considered for adjuvant chemotherapy or chemoradiation (category 2B) (10). Acknowledging depth of muscular invasion may be a useful prognostic factor to help identify who may optimally benefit from adjuvant therapy. We hypothesize that in patients with adenocarcinoma of the esophagus, tumor invasion into the outer longitudinal muscle layer correlates with reduced survival compared to invasion limited to the inner circular layer, after adjustment for nodal status and other high-risk features.

\section{Patients and Methods}

Institutional databases were used to identify patients with pT2N03M0 esophageal adenocarcinoma resected between 2005-2015 at Rush University Medical Center (Chicago, IL, USA), University of Wisconsin-Madison (Madison, WI, USA), Roswell Park Cancer Institute (Buffalo, NY, USA), and Mayo Clinic-Jacksonville (Jacksonville, FL, USA). Institutional Review Board approval was obtained at each participating institution (Rush University Medical Center: 14022402-IRB01; Roswell Park Cancer Institute: BDR71716; Northwestern Memorial: STU00202945; Mayo Clinic-Jacksonville: 15-007725; University of Wisconsin-Madison: 2015-0266)

Patients with pathologically confirmed T2N0-3M0 adenocarcinoma of the esophagus that underwent esophagectomy were included. Patients' electronic medical records and the Social Security Death Index were queried for demographic and oncological data including date of surgery, body mass index, gender, race, smoking history [current, never, ever ( $>100$ cigarettes ever)], preoperative comorbidities (chronic obstructive pulmonary disease, coronary artery disease, diabetes mellitus), operation status (elective or other), operation performed, R0 status, pathologic TNM stage, tumor grade (well-, moderately-, or poorly-differentiated), location (upper, middle, or lower third of esophagus, or gastroesophageal junction), tumor length $(<3 \mathrm{~cm}, 3-5 \mathrm{~cm}$, or $>5 \mathrm{~cm})$, administration of induction or adjuvant chemotherapy or chemoradiation, date of recurrence, and date of death or last follow-up.

Representative histological tumor specimens were chosen by an institutional pathologist (at institutions outside of Rush University Medical Center) that allowed for differentiation of circular and longitudinal muscle tumor invasion. Photomicrographs were electronically transmitted to Rush University Medical Center, where they were re-reviewed by a second pathologist (B.M.) who was blinded to all clinical and pathologic data. Depth of invasion and the presence of perineural invasion were re-evaluated. In all cases, there was agreement between pathologists regarding depth of invasion and the presence of perineural invasion. The primary study endpoint was OS with a secondary end point of DFS. Patient follow-up data were collected through May 1, 2016 at all participating sites. Patients younger than 18 years, with non-elective esophagectomy, noncurative (R1 or R2) resection, M1 disease, or death within 30 days of surgery or during index hospitalization were excluded.

Due to the multi-institutional nature, staging practices varied slightly, but patients routinely underwent positron-emission tomography scan and endoscopic ultrasound evaluation. Additionally, all patients had pathological confirmation of adenocarcinoma by endoscopic biopsy prior to treatment. Staging was performed according to the seventh edition of the AJCC cancer staging manual (8). All patients were reviewed by a multidisciplinary tumor board and treated per consensus recommendations. Operative technique was chosen according to surgeon judgment. Patients generally underwent follow-up imaging and surveillance in accordance with the NCCN guidelines (10).

Overall survival (OS) was calculated from the date of surgery to death or last documented follow-up. Disease-free survival (DFS) was calculated from the date of surgery to the date of first recurrence, defined as the last date of negative radiographic imaging and clinical examination.

Statistical analyses included chi-square tests and Fisher's exact tests for qualitative variables, correlation analyses, as well as twosample $t$-tests and Mann-Whitney tests of quantitative values between patients with circular and longitudinal muscle invasion. OS was estimated by the Kaplan-Meier method and was compared between groups by the log-rank test. The effect of depth of muscular invasion on survival after adjustments by other clinical predictors was assessed by multivariate Cox proportional hazards regression. A post-hoc analysis was performed to examine patients who underwent upfront surgery without induction therapy.

\section{Results}

A total of 84 patients (53 with circular invasion; 31 with longitudinal invasion) who underwent esophagectomy for pT2N0-3M0 esophageal adenocarcinoma met the inclusion criteria. The study group consisted primarily of Caucasian males, with a median age of 66 years, and tumors of the distal third or gastroesophageal junction (Table I). Most esophageal resections were performed in a minimally invasive fashion and were pathologically node-negative. There was no difference in age, gender, race, smoking history, comorbidities, tumor length, operation performed, perineural invasion, differentiation, or rate of induction therapy status between patients with circular and those with longitudinal muscle invasion. Although not statistically significant, $32 \%$ (17/53) of patients with circular muscle involvement had node-positive disease compared to $48 \%$ $(15 / 31)$ of those with longitudinal muscle invasion. Of the 34 patients who underwent surgery without neoadjuvant therapy, 14 (41\%) had pathologically node-positive disease, including six with circular invasion and eight with longitudinal invasion.

Overall, $50(60 \%)$ patients received neoadjuvant chemoradiotherapy $(n=48)$ or chemotherapy $(n=2)$. Of the 14 patients who underwent upfront surgery and had pathologically node-positive disease, $10(71 \%)$ received adjuvant therapy (seven chemoradiation; two chemotherapy only; one radiation only). There were 20 pT2N0 patients who underwent upfront surgery and none received adjuvant therapy. The median OS and DFS were 58 months [95\% confidence interval $(\mathrm{CI})=42$ months - not reached) and 27 months (95\% CI=13.7-66 months) for the entire cohort, 
Table I. Patient characteristics

\begin{tabular}{|c|c|c|c|c|}
\hline Characteristic & $\begin{array}{l}\text { All patients } \\
\quad(\mathrm{n}=84)\end{array}$ & $\begin{array}{c}\text { Circular invasion } \\
\qquad(\mathrm{n}=53)\end{array}$ & $\begin{array}{l}\text { Longitudinal invasion } \\
\qquad(\mathrm{n}=31)\end{array}$ & p-Value \\
\hline Median age (IQR), years; & $66(58-74)$ & $64(58-72)$ & $71(63-77)$ & 0.11 \\
\hline Gender, Male & $73(87)$ & $48(91)$ & $25(81)$ & 0.31 \\
\hline Median BMI (IQR), kg/m2 & $27.4(24-30.8)$ & $28.2(25.7-31.3)$ & $25.9(22.8-28.7)$ & 0.007 \\
\hline Race, Caucasian & $79(94)$ & $50(94)$ & $29(94)$ & 0.63 \\
\hline Smoker, n (\%) & & & & 0.95 \\
\hline Never & $20(24)$ & $12(23)$ & $7(23)$ & \\
\hline Past & $52(62)$ & $34(64)$ & $19(61)$ & \\
\hline Current & $12(14)$ & $7(13)$ & $5(16)$ & \\
\hline \multicolumn{5}{|l|}{ Comorbidity, n (\%) } \\
\hline COPD & $9(11)$ & $7(13)$ & $2(6)$ & 0.48 \\
\hline CAD & $26(31)$ & $18(34)$ & $8(26)$ & 0.68 \\
\hline $\mathrm{DM}$ & $22(26)$ & $14(26)$ & $8(26)$ & 0.97 \\
\hline Tumor Location, n (\%) & & & & 0.14 \\
\hline Middle 1/3 & $4(5)$ & $1(2)$ & $3(10)$ & \\
\hline Lower $1 / 3$ or GEJ & $80(95)$ & $52(98)$ & $28(90)$ & \\
\hline Tumor length, $\mathrm{n}(\%)$ & & & & 0.07 \\
\hline$<3 \mathrm{~cm}$ & $54(64)$ & $39(74)$ & $15(48)$ & \\
\hline $3-5 \mathrm{~cm}$ & $18(21)$ & $8(15)$ & $10(32)$ & \\
\hline$>5 \mathrm{~cm}$ & $12(14)$ & $6(11)$ & $6(19)$ & \\
\hline Operation performed, $\mathrm{n}(\%)$ & & & & 0.26 \\
\hline ILE & $22(26)$ & $16(30)$ & $6(19)$ & \\
\hline MIE & $39(46)$ & $25(47)$ & $14(45)$ & \\
\hline McKeown & $2(2)$ & 0 & $2(6)$ & \\
\hline THE & $20(24)$ & $11(21)$ & $9(29)$ & \\
\hline Thoracoabdominal & $1(1)$ & $1(2)$ & 0 & \\
\hline pT-stage, n (\%) & & & & $<0.001$ \\
\hline $\mathrm{T} 2$-circular & $53(63)$ & $53(100)$ & 0 & \\
\hline T2-longitudinal & $31(37)$ & 0 & $31(100)$ & \\
\hline pN-stage, n (\%) & & & & 0.16 \\
\hline No & $52(62)$ & $36(68)$ & $16(52)$ & \\
\hline N1 & $18(21)$ & $11(21)$ & $7(23)$ & \\
\hline $\mathrm{N} 2$ & $12(14)$ & $6(11)$ & $6(19)$ & \\
\hline N3 & $2(2)$ & 0 & $2(6)$ & \\
\hline Perineural invasion, $\mathrm{n}(\%)$ & $8(10)$ & $5(9)$ & $3(10)$ & $>0.99$ \\
\hline Differentiation, $\mathrm{n}(\%)$ & & & & 0.92 \\
\hline Well & $8(10)$ & $5(9)$ & $3(10)$ & \\
\hline Moderately & $45(54)$ & $28(53)$ & $17(55)$ & \\
\hline Poorly & $31(37)$ & $20(38)$ & $11(35)$ & \\
\hline Induction therapy, n (\%) & $50(60)$ & $32(60)$ & $18(58)$ & $>0.99$ \\
\hline
\end{tabular}

BMI, Body mass index; CAD, coronary artery disease; COPD, chronic obstructive pulmonary disease; DM, diabetes mellitus; GEJ, gastroesophageal junction; ILE, Ivor Lewis esophagectomy; IQR, interquartile range; MIE, minimally-invasive esophagectomy; THE, transhiatal esophagectomy.

respectively, with a median follow up of 15.1 months (interquartile range=7.6-25.5 months). For those who underwent upfront surgery, the median OS and DFS were 65.5 months $(95 \% \mathrm{CI}=41.9$ months-not reached) and 65.5 months (95\% CI=27.2 months-not reached), respectively.

In univariate analysis, no demographic or pathological characteristics were associated with OS in the study cohort $(n=84)$, nor in the subgroup managed with upfront surgery $(n=34)$ (Table II). In multivariate analysis, perineural invasion was identified as a poor prognosticator of OS (hazard ratio $(\mathrm{HR})=5.19,95 \% \mathrm{CI}=1.27-21.25 ; p=0.02]$ in the study cohort $(n=84)$. In patients who underwent upfront surgery $(n=34)$, no demographic or pathologic characteristics were associated with OS in the multivariate analysis. Perineural invasion could not be analyzed in the upfront surgery multivariate model due to the limited number of events. Depth of esophageal muscularis propria invasion (circular or longitudinal layer) was not associated with OS in the study cohort (Figure 1A), nor in those who underwent upfront surgery (Figure 1B), after controlling for other high-risk factors (age, $\mathrm{pN}$-status, perineural invasion, and tumor grade). 
Table II. Univariate and multivariate predictors of overall survival.

\begin{tabular}{|c|c|c|c|c|c|c|}
\hline \multirow[b]{3}{*}{ Factor } & \multicolumn{3}{|c|}{ All patients $(n=84)$} & \multicolumn{3}{|c|}{ Upfront surgery $(\mathrm{n}=34)$} \\
\hline & \multirow[b]{2}{*}{ Univariate $p$-Value } & \multicolumn{2}{|c|}{ Multivariate } & \multirow[b]{2}{*}{ Univariate $p$-Value } & \multicolumn{2}{|c|}{ Multivariate } \\
\hline & & $\operatorname{HR}(95 \% \mathrm{CI})$ & $p$-Value & & HR $(95 \% \mathrm{CI})$ & $p$-Value \\
\hline Age & 0.88 & $1.01(0.97-1.05)$ & 0.62 & 0.51 & $1.01(0.91-1.11)$ & 0.89 \\
\hline pT circular invasion & 0.24 & $0.50(0.20-1.27)$ & 0.15 & 0.66 & $1.99(0.23-17.04)$ & 0.53 \\
\hline pN-positive & 0.56 & $2.02(0.82-4.97)$ & 0.13 & 0.72 & $1.95(0.32-12.02)$ & 0.47 \\
\hline Poorly differentiated & 0.49 & $2.14(0.88-5.20)$ & 0.09 & 0.76 & $0.34(0.02-5.70)$ & 0.45 \\
\hline Perineural invasion & 0.29 & $5.19(1.27-21.25)$ & 0.02 & 0.50 & & \\
\hline Gender & 0.07 & & & 0.37 & & \\
\hline BMI & 0.31 & & & 0.16 & & \\
\hline Smoker & 0.64 & & & 0.69 & & \\
\hline Tumor location & 0.50 & & & 0.76 & & \\
\hline Tumor length & 0.15 & & & 0.06 & & \\
\hline
\end{tabular}

BMI, Body mass index.

Table III. Univariate and multivariate predictors of disease free survival.

\begin{tabular}{|c|c|c|c|c|c|c|}
\hline \multirow[b]{3}{*}{ Factor } & \multicolumn{3}{|c|}{ All patients $(n=84)$} & \multicolumn{3}{|c|}{ Upfront surgery $(\mathrm{n}=34)$} \\
\hline & \multirow[b]{2}{*}{ Univariate $p$-Value } & \multicolumn{2}{|c|}{ Multivariate } & \multirow[b]{2}{*}{ Univariate $p$-Value } & \multicolumn{2}{|c|}{ Multivariate } \\
\hline & & HR $(95 \% \mathrm{CI})$ & $p$-Value & & $\mathrm{HR}(95 \% \mathrm{CI})$ & $p$-Value \\
\hline Age & 0.68 & $1.0(0.97-1.03)$ & 0.99 & 0.39 & $1.0(0.93-1.08)$ & 0.95 \\
\hline pT circular invasion & 0.34 & $0.64(0.31-1.29)$ & 0.21 & 0.21 & $2.38(0.47-12.03)$ & 0.30 \\
\hline pN-positive & 0.12 & $2.45(1.21-4.98)$ & 0.01 & 0.69 & $1.47(0.32-6.83)$ & 0.63 \\
\hline Poorly differentiated & 0.30 & $1.59(0.79-3.22)$ & 0.19 & 0.79 & $0.56(0.08-3.76)$ & 0.55 \\
\hline Perineural invasion & 0.11 & $4.75(1.57-14.41)$ & 0.01 & 0.43 & & \\
\hline Gender & 0.36 & & & 0.92 & & \\
\hline BMI & 0.08 & & & 0.08 & & \\
\hline Smoker & 0.39 & & & 0.75 & & \\
\hline Tumor location & 0.35 & & & 0.66 & & \\
\hline Tumor length & 0.55 & & & 0.001 & & \\
\hline
\end{tabular}

BMI, Body mass index.

Tumor length was independently associated with DFS in univariate analysis of patients who underwent upfront surgery $(p=0.001)$. However, no other demographic or pathological characteristics were associated with DFS in the study cohort in univariate analysis (Table III). In the multivariate model, perineural invasion was found to be a poor prognosticator of DFS ( $\mathrm{HR}=4.75,95 \% \mathrm{CI}=1.57-14.41$; $p=0.01)$. In patients who underwent upfront surgery, no demographic or pathological characteristics were associated with DFS in multivariate analysis. Again, perineural invasion could not be analyzed due to the limited number of events. Depth of tumor invasion into the muscularis propria (circular or longitudinal layer) was not associated with DFS in the study cohort (Figure 2A) nor in those who underwent upfront sugery (Figure 2B) after controlling for high-risk factors including age, $\mathrm{pN}$-status, perineural invasion, and tumor grade.

\section{Discussion}

It is well-established that the prognosis of esophageal cancer worsens as the depth of tumor invasion increases. Despite this, the seventh edition of the AJCC staging system does not differentiate between invasion into the circular or longitudinal muscular layers of the muscularis propria, a characteristic that is distinguishable by pathological analysis 
A

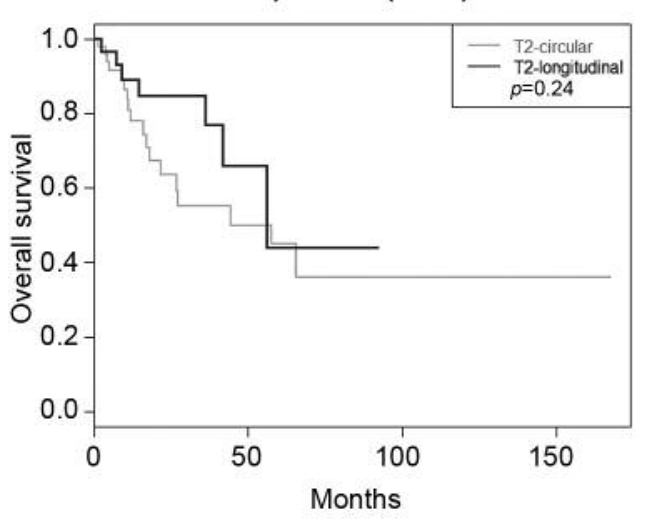

\section{B No induction therapy $(n=34)$}

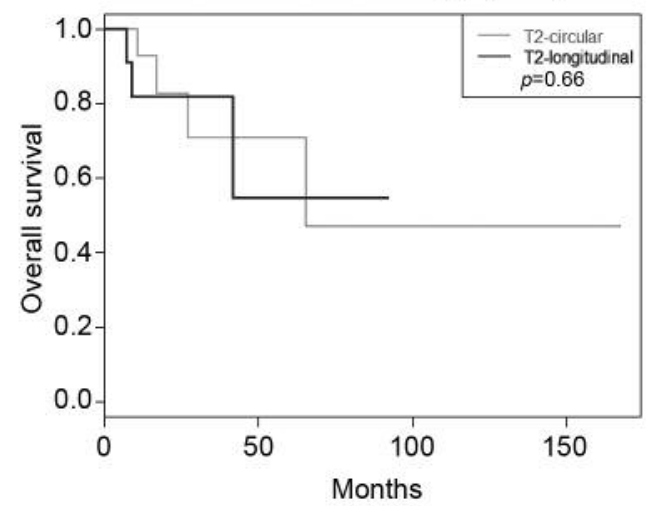

Figure 1. Overall survival by depth of tumor invasion in the entire cohort (A) and those who underwent upfront surgery without induction therapy (B).

A

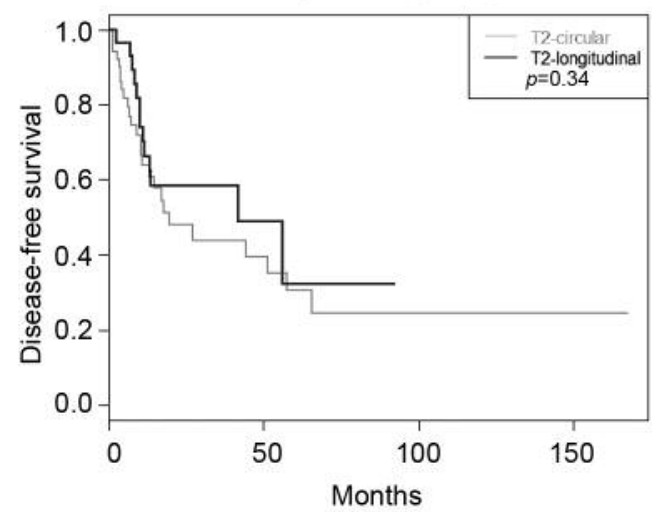

B No induction therapy $(n=34)$

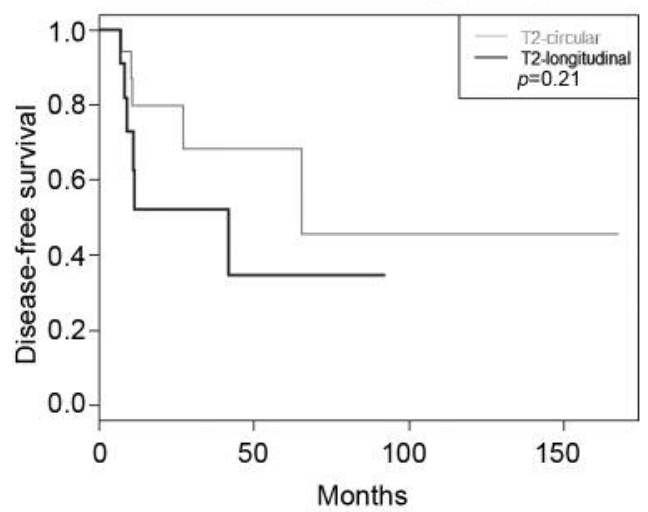

Figure 2. Disease-free survival by depth of tumor invasion in the entire cohort (A) and those who underwent upfront surgery without induction therapy $(B)$.

(8). Recently, a study of 85 patients with pathologic T2N0 esophageal squamous cell carcinoma identified that tumor invasion limited to the inner circular muscle layer was associated with superior OS compared to invasion into the outer longitudinal layer, after controlling for high-risk features (9). However, the current multi-institutional study was unable to demonstrate an association between depth of invasion and survival in patients with esophageal adenocarcinoma, after controlling for age, nodal status, perineural invasion, and tumor grade.

Given that esophageal adenocarcinoma originates in the mucosa, it is conceivable that depth of tumor invasion into the muscularis propria may be an important prognostic marker. Such a marker may re-define staging classifications and therapeutic strategies, modify indications for adjuvant therapy, and impact recommendations for surveillance. According to the NCCN guidelines, select 'high-risk' patients with pT2N0 esophageal adenocarcinoma may benefit from adjuvant chemoradiation (level 2B) (10). Within these guidelines, high-risk features include poorly differentiated tumor, perineural invasion, or age less than 50 years. If tumor invasion into the longitudinal layer of the muscularis propria were found to be a poor prognostic indicator, it might provide valuable information for future refinements of the AJCC staging system. The current data demonstrated no association between depth of invasion and survival; however, the limited number of patients with pT2N0 disease $(n=20 ; 15$ with circular invasion, five with longitudinal invasion) who underwent upfront surgery made a post-hoc survival analysis difficult to interpret. 
Current NCCN guidelines do not provide category 1 recommendations for the postoperative management of patients with pathological $\mathrm{T} 2$ esophageal adenocarcinoma treated with upfront surgery (10), however these patients are often managed with fluoropyrimidine-based chemoradiation for node-positive or high-risk, node-negative patients. The data presented in this analysis from four geographically diverse, academic, tertiary-care centers reflect modern trends in treatment of this esophageal cancer subgroup, with $71 \%$ of patients with node-positive disease receiving adjuvant therapy, but none of those with node-negative disease.

These data suggest that depth of muscularis propria invasion is not an independent predictor of OS or DFS. Perhaps it is not surprising that our findings are discrepant from previously reported data in esophageal squamous cell carcinoma (9). Esophageal squamous cell carcinoma and adenocarcinoma occur largely in different patient populations, have a divergent pathogenesis and tumor behavior, and are staged using separate classifications. However, the fact that nodal status, a widely accepted and routinely utilized metric in the staging of nearly all malignancies, did not prognosticate survival is counterintuitive. These conclusions suggest that additional factors not explored in this analysis, such as genetic mutations, patient comorbidities, or patient selection bias may have had a significant impact on survival.

A more likely explanation for the discrepant findings of this study relates to stage migration and the relatively small sample size. A patient with a clinical T3N2M0 esophageal adenocarcinoma who receives induction therapy, and whose disease is effectively down-staged to a pT2N0 tumor, does not have the same disease process as a patient managed with upfront esophagectomy for a clinical and pathological T2N0 adenocarcinoma. To address this, we performed a post-hoc analysis of patients who underwent upfront surgery. The relative rarity of patients with induction therapy-naïve pT2N0 esophageal adenocarcinoma resulted in a relatively small subgroup for analysis, making statistical significance difficult to demonstrate.

Limitations of the current study include the relatively short median follow-up and biases inherent to the retrospective study design. However, this analysis highlights the feasibility of a multi-institutional, surgeon-driven, collaborative effort to study a rare disease process, performed outside the confines of a clinical trial. This includes procurement of patient-level data that is not possible when large administrative databases are queried. As a result of its multi-institutional design, there was likely slight variation in the work-up, surgical technique, induction and adjuvant therapy regimens, and follow-up protocols that may have influenced survival. On the other hand, the multiinstitutional nature of these data increases the external validity and reflects current national trends in the use of adjuvant therapy for pT2N0-3M0 esophageal adenocarcinoma. Finally, only all-cause mortality, not cancer-specific mortality, was evaluated due to the availability of data.

In conclusion, unlike esophageal squamous cell carcinoma, the depth of invasion of the muscularis propria does not correlate with survival in patients with esophageal adenocarcinoma. Future studies with larger sample sizes and prolonged follow-up are required to validate these findings. Additional subgroup analyses focusing on the association between induction or adjuvant therapy and depth of muscle invasion will likely also yield clinically relevant data. This study highlights the feasibility of surgeons to work in a multiinstitutional fashion outside the confines of a clinical trial in order to examine clinically relevant management algorithms.

\section{References}

1 Ferlay J, Shin HR, Bray F, Forman D, Mathers C and Parkin DM: Estimates of worldwide burden of cancer in 2008: GLOBOCAN 2008. Int J Cancer 127: 2893-2917, 2010.

2 Jemal A, Siegel R, Ward E, Hao Y, Xu J and Thun MJ: Cancer statistics, 2009. CA Cancer J Clin 59: 225-249, 2009.

3 Lepage C, Rachet B, Jooste V, Faivre J and Coleman MP: Continuing rapid increase in esophageal adenocarcinoma in England and Wales. Am J Gastroenterol 103: 2694-2699, 2008.

4 Rice TW, Mason DP, Murthy SC, Zuccaro G Jr., Adelstein DJ, Rybicki LA and Blackstone EH: T2N0M0 esophageal cancer. J Thorac Cardiovasc Surg 133: 317-324, 2007.

5 Kutup A, Link BC, Schurr PG, Strate T, Kaifi JT, Bubenheim M, Seewald S, Yekebas EF, Soehendra N and Izbicki JR: Quality control of endoscopic ultrasound in preoperative staging of esophageal cancer. Endoscopy 39: 715-719, 2007.

6 Pech O, Gunter E, Dusemund F, Origer J, Lorenz D, Ell C: Accuracy of endoscopic ultrasound in preoperative staging of esophageal cancer: results from a referral center for early esophageal cancer. Endoscopy 42: 456-461, 2010.

7 Bergeron EJ, Lin J, Chang AC, Orringer MB, Reddy RM: Endoscopic ultrasound is inadequate to determine which T1/T2 esophageal tumors are candidates for endoluminal therapies. J Thorac Cardiovasc Surg 147(2): 765-773, 2014.

8 Rice TW, Blackstone EH, Rusch VW: 7th edition of the AJCC Cancer Staging Manual: esophagus and esophagogastric junction. Ann Surg Oncol 17: 1721-1724, 2010.

9 Guo W, Xiao HL, Ma Z, Liu HJ, Wang YJ, Mei LY, Liu XH, Jiang YG and Wang RW: Should stage T2 esophageal squamous cell carcinoma be subclassified? Ann Surg Onc 21: 2540-2545, 2014.

10 NCCN Clinical Practice Guidelines in Oncology: Esophageal Cancers and Esophagogastric Junction (Excluding the proximal $5 \mathrm{~cm}$ of the stomach); Version 2.2016. https://www.nccn.org/ professionals/physician_gls/pdf/esophageal.pdf Accessed February 20, 2017.
Received January 22, 2018

Revised February 13, 2018 Accepted February 21, 2018 